cases there have been slight recurrences, small nodules appearing at the margins, and, as already mentioned, these are probably due to the escape of small outlying parts from the action of the rays. In some, recurrences have been seen more than once. As a rule, however, these recrudescence are easily dealt with by a fresh application of the treatment.

It is very difficult to give any accurate idea as to the time which a given case will have to be subjected to the rays. This is an important consideration with many patients. I have had small ulcers which have entirely healed with from ten to a dozen exposures; but where there are large cavities, and especially where there is a very tough, nodular edge, month may be required. The points considered in the preceding paragraph indicate the special difficulties.

I am accustomed to apply the rays on alternate days in the slighter cases, and daily in the more extensive ones. still use tubes which spark at from 4 to 6 in., but I find that the therapeutic value of the tubes is a very variable thing, and one which at present it is impossible to explain. "High" tubes are valuable, as the rays can be applied until there is a definite inflammatory reaction. Actual "burning" is not necessary, and some of the best resalts have been obtained without any obvious inflammatory reaction at all. I protect the surrounding skin with leaden masks, but I have used Cosser's tubes with advantage, but find that they can rarely stand heavy out-patient work, as at the London Hospital, where the tubes are worked for several hours daily

I have no hesitation in saying that the $x$ rays still hold the field as the best therapeutic agent for the treatment of rodent ulcer in those cases in which complete excision of the growth cannot be carried out. The following are details of two interesting cases:

CASE I.-T. W. R., aged 55, a draper's assistant. For eighteen years the patient had suffered from rodent ulcer. The disease began at the right ala nasi and slowly extended. It was diagnosed as lupus, and several operations were done in New Zealand. The upper lip, the nose, and finally a large part of the upper jaw and palate were involved and removed by operation. The result was the formation of a hideous cavity in the middle of the face, the base of the ulceration being irregular, and exuding a foul, purulent discharge. The unfortunate patient was obliged to wear a black silk mask to hide his deformity. The eondition is shown in Fig. $x$. The patient was under treatment for nearly nine months. The rays were applied for ten minutes a day, and with but short periods of rest. The ulceration healed up in a remarkable manner, and a large part of the cavity flled up. The condition after the $x$-ray treatment is shown in Fig. 2. As soon as the scar was sound I called my colleague, Mr. Frank Farmer, Dental Surgeon to the London Hospital, into consultation, and he undertook to try and repair the deformity. He first fitted a plate to take the place of the upper jaw and palate, and when this was in position the patient was able to swallow and to masticate. After this a false nose with an extension for the upper lip was made, and a false moustache added, and the result proved most satisfactory. The condition of the patient when he left Mr. Farmer's hands is seen in Fig. 3. Th patient returned to New Zealand, and when last heard of was still well.

CAFE II. - The patient, a woman aged ${ }_{3 I}$, had suffered from the disease for nineteen years. It thus began at the age of 12 , and is, I believe, the earliest on record. The diagnosis of rodent ulcer was obvious from the appearance of the ulcer, with its hard, raised, nodular edge, which are well shown (Fig. 4). It was verified by the microscopical examination of a portion excised before treatment was begun. In this case the ulcer started as a small warty growth at begun. In ala nasi, and gradually extended. No operation had been performed. The ulcerated area soon healed under the rays, but the hard rolled edge proved very resistant, and was treated at intervals for six months. Applieations were made about three times a week. The hard edge entirely disappeared (Fig. 5). The patient has been free from recurrence for six months. The dark colour of the scar is due to pigmentation which has almost disappeared since the photograph was taken.

The comparison of these two cases is very instructive. Both started at the right ala nasi, and both had lasted nearly twenty years before coming under the $x$-ray treatment. In one frequent operations had been performed, with the result that hideous deformity had been produced. In the other no operation had been performed, and the result is excellent. A case under my care in which there had been no recurrence for over two years is figured in the Medical Annual for 1903.

Rkgistration of Trained NuRses in AMERICA.-The Governor of New York State has signed the Bill passed by the Legislature providing for the registration of trained nurses in the State, under the auspices of a Board of Examiners to be appointed by the regents of the University of the State of New York. Nurses passed by the Board, and certain nurses qualified by long service prior to the passage of this law, are entitled to the designation of " registered nurse," and to attach the initials "R.N." to their names. The registration of nurses has also been adopted by the States of North Carolina, Illinois; New Jersey, and Virginia.
TREATMENT OF LUPUS BY $X$ RAYS AND ULTRAVIOLET RAYS.

By H. E. GAMLEN, M.B., B.S., D.P.H., West Hartlepool.

IT is highly important that the results of the $x$-ray treatment should be emphasized by full and detailed publication. There is undoubted evidence that the benefit to be derived from $x$ rays is almost illimitable - the great aim of those who adopt this method of treatment is to endeavour to utilize to the fullest the healing qualities and power of the rays. Keen observation and sound judgement are essential, and I have no hesitation in asserting that the success attained is in proportion to the exercise of these faculties. The results already achieved are of an excellence and endurance sufficient to give substantial indication of what one may expect from the rays in-let us hope-the near future.

$X$ Rays and Operation Compared.

The advantage of $x$ rays over operative treatment are principally cosmetic in effect. After healing they leave a soft pliable skin devoid of contractions, and the face is not disfigured by the unsightly scars which are the inevitable consequence of the use of the knife. The risk from anaesthetic is obviated, and the patient cured without pain or shock. It is an established fact that in many skin diseases the application of the $x$ rays abolishes the necessity for operation. Those under treatment can also at the same time carry on their ordinary avocations. Many who fear the danger of an operation hesitate, and in this way the disease is spread and becomes more difficult to cope with. In $x$ rays, however, a discovery has been made which entirely overcomes this objection.

This is a general and broad view to take of the question, and it becomes necessary to particularize in order to form some guidance in $x$-ray treatment. With this object in view I have quoted below twelve cases of lupus successfully treated by me. From my experience of these and many others, together with cases of cancer, rodent ulcer, lupus ery thematosus, and various skin diseases, curative treatment is dependent, upon earnest consideration of the following points : The character of coil; the voltage used; the condition of the tubes; the distance of the target from the patient; the duration and frequency of sittings with the interval between each; and finally, whether or not any other treatment is concurrently applied.

\section{Attention to Details.}

These are details too often overlooked, but they are of vital importance in the attainment of the end desired. No hardand-fast rule can be adopted, but I have come to the conclusion that the effect of the rays can be applied to individual cases and regulated to suit stages of disease by strict attention to the injunctions enumerated. This applies more especially to the condition of tubes and the limitation or extension, according to requirements, of the exposures given to patients. At the same time full notes should be tuken, for the worries and anxieties of a busy practitioner do not admit of an infallible memory. I have found that careful observation, attention to detail and a close study of each case are the greatest factors in successful treatment. For instance, I frequently test the variety of tubes at my disposal, and am, therefore, always well acquainted with their condition and capabilities.

There are many who place too great reliance in the length of the sitting. In many articles $I$ have read the period of exposures adopted by the writers was stated to be from ten minutes to an hour every day. This theory has not held good with me. It has always been my object to obtain rapidly a tangible result consistent with safe treatment. Instead of daily exposures I have adopted a system of giving sittings two or three times a week. lasting from three to five minutes. The brevity of the period I attribute to the fact that I use a 14-in. coil, and always obtain an ampèrage of from six to eight, and a forty-volt pressure. When beginning treatment it is impossible to exercise too much care. At this stage I always use a low tube-and then watch the case. The primary object is to produce a mild reaction. Perhaps it may be necessary for this purpose to use a still lower tube, and increase the length of the sitting. This is where the faculty of judgement enters into the treatment, and when the individual necessities of the patients must be considered.

I am firmly convinced that in order to cure, a mild reaction 
must at the outset be promoted, and then steadily, and even skilfully maintained. The extent of this reaction must be determined slowly by discretion in the regulation of tubes and exposures. Once having found a tube to suit a particular case I obtain the desired effect by increasing or decreasing the interval and length of sittings, the distance between the patient and the target, and at the same time controlling the current through the coil. In obstinate cases which do not respond readily to treatment $I$ use lower degrees of tubes until I have succeeded in producing a sufficiently severe reaction. Then I suspend the sittings for three or four days or until the result of the exposure is apparent. Upon this I base the course of future treatment. In the majority of cases the dermatitis has become evident within two or three dayssomewhat less than the experience of others. The subsequent healing process is tolerably speedy, and the skin, formerly diseased, then assumes a smooth appearance. The severity of the reaction necessary, however, must always depend upon the character of the case and the susceptibility of the patient under treatment. I am greatly in favour of the use of low resisting tubes for superficial surfaces. For this purpose have compared them with the hard tubes, and find that if judiciously regulated they give more speedy and sure results. Where penetration is necessary, as for deep-seated ulceration, tubes of a hard nature are undoubtedly the most efficacious. This is perhaps the only theory approaching generality which I would be prepared to propound. For instance some tubes apparently the same in resistance and spark length produce different effects. The obvious deduction to be drawn is that complete success is to a large extent attributable to a varied supply of tubes, the capabilities of which are well-known and recorded.

In dealing with some cases I combined the ultra-violet rays (lupus lamp) with the $x$ rays. I have also tried the lupus lamp (Leslie Miller) alone. With the ultra-violet rays there is ver little subsequent aiscoloration in the situation of the surround. ing tissues. 'The same does not apply to the $x$ rays, the pigmentation being more apparent when low tubes are used. I am of opinion that for small areas the lupus lamp is most effective, but the following conditions must be complied with: Smear the affected parts with vaseline, uge ice, and apply adequate pressure. The use of vaseline appears to increase the value of the light. I never give any accessury treatment with either the lupus lamp or $x$ rays. One point upon which I would insist is the necessity for personal supervision by medical practitioners. The apparatus should never be left in charge of incompetent persons, for without constant observation and strict attention to detail the fullest possible benefit cannot be derived.

The lupus cases which I quote below are taken at random from my list. I have always succeeded in effecting a complete cure, and the method adopted is fully explained. I trust that this contribution will lead other practitioners to relate their experiences, for on such a subject, when there is so much to learn details are in great demand. Nevertheless, the cases mentioned may be regarded as typical. Some of them were obstinate, whilst other yielded readily to treatment. That proves at least that no definite procedure can be adoptedmuch must depend upon the patient and the practitioner. It is too early yet to assert definitely that the cures will be permanent-even operation does not ensure that. There is good reason to believe, however, that the disease will not recur and the patients at least are well satisfied with the relief attained, even presuming it were only temporary.

CASE 1-A woman, aged 54. Duration of disease four years. The nose and both sides of the face were a mass of lupus, extending on the right cheek to just below the eyelid, beneath which the ravages of the disease had left a deep cavity. The upper lip and interior of both nostrils were affected, but there was no perforation. In 1809 I operated three times finally under an anaesthetic, when I used the knife and spoon freely, and also grafted healthy skin where necessary. Six months later the patient returned, and the only portion cured was that beneath the eyelid. This was denoted by a well-marked scar. The remaining lupus tracts were in a worse condition than before the operation, the passages of both nostrils worse condition than before the operation, the passages of both nostrils
being entirely closed with lupus nodules and the resultant inflammation Subsequently she had recourse to "quack" remedies, but again visited mein November, roor. During the interval the disease had developed so rapidly that her nose was now very much swollen, and, together with her lips, was covered with ulcerated tubercles which bled freely when touched. I then gave her $x$-ray sittings of five minutes' duration twice a weekfourteen in all. From the first the pain about which the patient had comfourteen in all. From the first the pain about which the patient had comset in. On January roth, 1902, I increased the sitting to ten minutes, bu owing to the dermatitis which followed, the treatment was interrupted for eleven days. On the ruth I made an examination; the nose.was again very much swollen, tender, and painful. On the 2 rst, however, there was no sign of lupus on the nostril and upper lip, but the interior of the nostril was still affected. I also noticed that the soar under the eyelid was not so prominent. I recommenced the $x$-ray treatment with fiveminute sittings, twice a week, until the end of February, when all traces of the disease seemed to have been eradicated. she came back once more on March zoth, roo2, in consequence of a slight return of the lupus at the of the skin had formerly been diseased were blanched and pigmented. The scar under the right eyelid had practically gone. Only four more sittings were considered necessary. At the end of October, 1902, a small spotalmost microscopical in appearance-recurred on the right side. This is now under treatment, and there is no doubt that after a few applications it will depart entirely and so complete the cure. With the single and slight exception referred to, the place has resumed a normal appearance. To a large extent attribute this exceedingly satisfactory result to the reaction prompted by a low tube and extended sitting.

Case II.-A woman, aged 50 . Duration of disease ten years. Four years ago the patient was subjected to mercurial treatment for a year. No improvement resulted, and she then came to me. This was about two years ago, and she was under my treatment for a year. I could find no specife history to justify mercurial treatment. Both cheeks had been affected, the lupus spreading over the lower part of the nostrils, inside each of which was a mass of nodules. The result was perforation of the septum to such an extent as to admit the small finger. I operated four times in 9or, thoroughly scraping and touching the parts with pure carbolic acid. anasthetic. again removed the diseased tissues with spoon and anaesthetic. aral day four months the lupus reappeared, and a month later was even more severe and the lupus reappeared the patient to $x$-ray treatment from August to the end of October, rэo2. She had five-minute sittings three times a week, the target of a fairly low tube being placed 9 in. from the face. A ro-in. coil was used, with a voltage of twelve, and ampèrage of from six to eight. After fourteen exposures no improvement was shown, but when another ten had been given, the nodules disappeared, with the exception of those in the mucous membrane in the interior of the nostril. With another twelve sittings all traces of lupus, however, vanished. The skin over the former unhealthy portions became blanched, smooth and soft with slight pigmentation, but no evidences of scar. There has been no recurrence and the hours before the rays.

CASE III.-A girl, aged 18. Duration of disease seven or eight years. A lupus patch about an inch in circumference in the centre of the right cheek. This patient had been twice operated on before coming under my treatment. In March, Igoo, I removed the lupus with the knife, making deep incision well clear of the growth, and grafted the cut surface. Ten mo recurred and was made additionally uusightly by months later the luring April, May, and June of roor I gave her twentythe large scar. During April, May, and June of rgor I gave her twenty five $x$-ray sittings of five minutes duration each. By this time the disease had practically disappeared. I then had recourse to a very low vacuum tube, but after the fourth application severe dermatitis ensued. This involved the loss of some hair. The healing process occupied a month. Then the hair commenced to grow again, and the lupus entirely vanished. There has been no recurrence, and it is now over a year since the patient was discharged cured after my treatment. There is no scar to the pation cardis be distinguished be seen, and the portion formerly affected can hardly be distinguished pigmentation.

CASE IV,-A woman aged 40. Thirteen years ago lupus commenced with a small nodule on the tip of the nose. About seven years ago the part was scraped. The disease returned a year later, and extended over the wis me roof of the mouth was also attacked. The oneration of scraping was again carried out. Six months later the dis operation of scraping was again carried out. Six matient went to a ase recurred more severely, and four years ago the patient went to gums, roof of the mouth, nose, and affected parts of the face were for the gurient visited me (Fig. $x$ ) third tim half of the bridge of the nostrils were covered with nodules, extending to the left side of the face. cheek. and upper lip, with ulcerations and crusts. The mucous membranes in both nostrils were covered with tubercular ulceration, and the septum was consequently perforated. The roof of the mouth presented a mass of tuberculous tissue, and in two situations the bare bone was displayed. The gums and the upper jaw were dotted with tuberculous ulceration. Both tear ducts were obstructed, and tears with tubereulous ultra-violet rays (Leslie ran over the face. In this case $I$ combined the patient ten-minute sittings Miller lupus lamp)'with the $x$ rays. I gave the patient ten-minute sittings twice a week with the lupus lamp-six in all. A great improvement was then noticeable. All the scabs dropped off, and were replaced by a healthy epithelium. The tuberculous tissue of the skin had disappeared, but the inside of the postrils, the gums, and the roof of the mouth were anchanged. I then treated her with the $x$ rays from a $x_{4}$-in. coil. I gave unchanged. If the outset I used low tubes, but found that this was. responsible for too severe reaction. I substituted a higher tube, and the results then attained were exceedingly satisfactory (Fig. 2). At the end of August, rgo2, the passage of the tear duct was free, and tears could now pass into the nostrils. The ulceration of the nostrils had, of course, been eradicated, but the gums and mouth were still affected. The improvement was, however, maintained. I then were still affected. The imper tube to focus the roof of the mouth, and utilized a specially-constructed tube to focus the roof of the mouth, and the case can be regarded as genuinely cured. The place of perforation in 
curient, and this is now perceptibly smaller. The same patient was suffering from tuberculous periostitis and osteitis of the tai sal bones. causing some lameness. Concurrent with the lupus treatment I gave exposures for th; foot, using a hard tube. All y ain vanished, the swelling abated. and the parts l esumed normal shape and rondition.

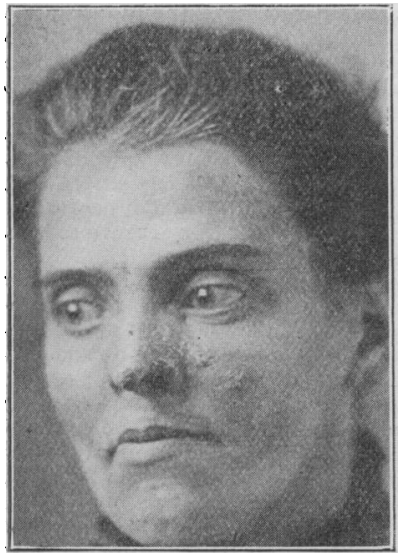

Fig. r.-Case iv; condition on March isth, 1902 .

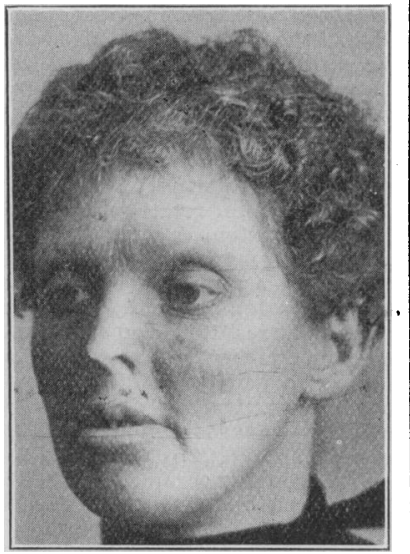

Fig. 2.-Case Iv : condition, on August $2=n d$, after four hours' applicatio 1 oi $x$
It may be of interest to add that this patient's brother was under the high-frequency current treatment for phthisis. A great improvement resulted. I treated another brother for a tuberculous wrist. which had been operated upon at a large infirmary in the North. The scar was visible and had broken down, ltading by a fistulous opening to an abscess in the wrist. Ait $r$ tivelve applications the disease was eliminated, and the patient, who had been out of employmeut six months, was able to resume work. It is a coincidence that another relative has heen nnder wy treatment for lupus.

CASE v.-Boy, aged 15. Duration of disease four years. From May until August, 18,8, was unde ${ }^{\circ}$ uedical treatment: subsequently admitted to an infirmary from October to November, and underwent an operation - scraping. A renewal occurred in May, 1899. The patient was admitted to another hospital, but eventually visited me on Apıil 25 th, 1902. (Fig. 3.) to another hospital, but eventually visited me on April 25 th, 1902. (Fig. 3.) nodul nodules. The tips of both alae were eaten away, and the disease had
extended partially ou to the upper lip, the inside of which, together with the gums surrounding the first four front teeth, were ulcerated. A alight portion of the hard palate was also attacked and ulcerated. The patient presented a most unsightlo appearance, and had been in the habit of wearing $\mathrm{d}$ b five to en minute sittings with the ultra-violet lamp. After twenty exposures the nose and upper lip yielded to treatment. The reason who the

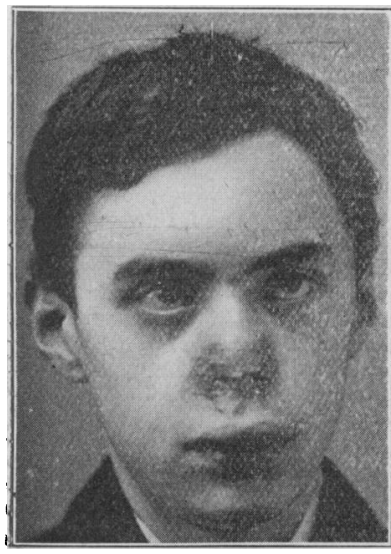
April 25th, 1902 .
Fig 3-Case $\mathrm{v}$; condition on

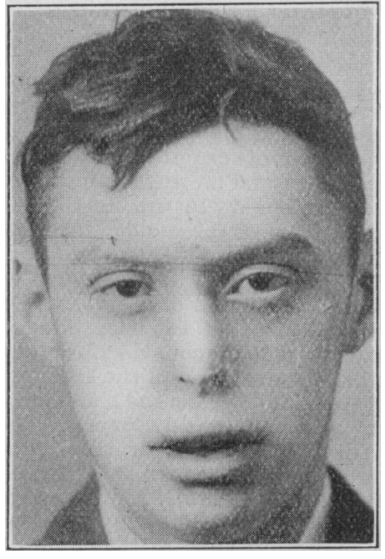

Fig. 4.-Case v: condition on October 24 th, aller tive hours' appli. cation of $x$. time taken did not compare faro urably with other cases was the reluctance of the patient to allow pressure to be applied as firmly as was essential. I next used the $x$ rays- $y_{4}$-in. coil-and fooussed the interior of the mouth with a special tube. On one occasiod a low vacuum tube and an extra long sitting caused a reactiois in the form of swelling of the upper lip, gums, and roof of the mouth. Treatment was consequently interrupted for about a fortnight, and when the swelling had abated I found that the facial appearance had materially improved. With this experience to guide me I then used "a fairly low tube, n.hich had a ten- dency to maintain a necessary reaction. Although the mouth and nostril were being focussed the remainder of the affected area received some proportion of the rass. The total number of sittings was fifty, and the patient may now be regarded as entirely cured (Fig. 4). Of course, it i too early to pronounce permanency, but there is every reason to believe that the disease will not return. The patient is able to obtain employment-a practical result of the treatment.

CASE vi.-A man, aged 35 . Duration of disease, several years. Description : Three large patches. One of these measured 3 in. by 3 in., and extended from the left ear over the side of the jaw and down to the upper neck. There was also a smaller patch on the right side, and another $x$ in. by $\frac{1}{2}$ in. on the cheek to the right of the nostril. On March 18 th rgo2, treatment commenced with the lupus lamp. After ten sittings the left side improved but the right did not progress satisfactorily. I then substituted the $x$ rays, and in this way treated a larger area than hitherto. I always used a low tube ind order to cause some reaction, and gave sittings at the outset once a week and then twice a week. Some excoria tion was noticeable, but eventually a complete cure was effected. In the place of raised scars the skin became supple and pliable, and resumed normal appearance. In this case slight dermatit is was regularly and purposely caused by a soft tube, but always healed in a week's time and left a tangible improvement.

CASE VII-A woman, aged 40. Duration of disease ten zears. The affected parts had been scraped and cauterized on thirteen different occasions. The whole of the sides and end of the nose were a mass of tuberculous nodules which bled freely and were covered with scalus. The interior of the nostrils showed slight ulceration. The patient came under my treatment on April ${ }_{4}$ th, 1902 . (Fig. 5.) I gave her sittings on alternate days. Twelve exposures with the $x$ rays lasting three minutes did not seem to have the desired effect. Then on two occasions I increased the limit to ten minutes, visg a low vacum tube. Following the course

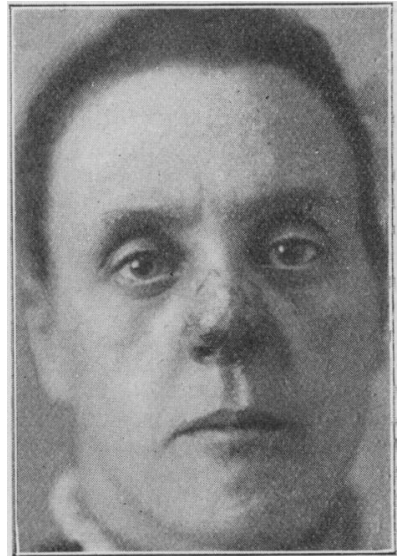

Fıg. 5.-Case vil a; condition ou April 4 th, 1502 .

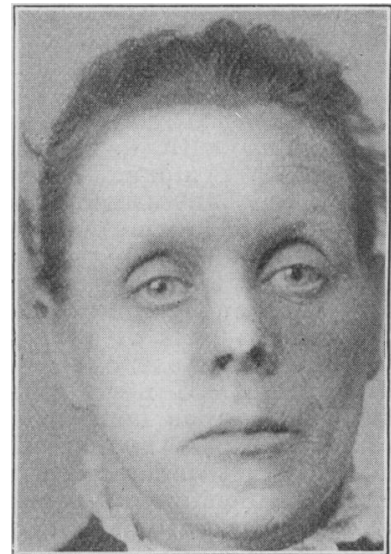

Fig. 6.-Case vil a; condition on August 2oth, 1902, after four hours rays combined. application of $x$ and ultra-violet

which I had always found most effective I purposely created a reaction. Owing to the severity of this I had some difficulty in persuading the patient to continue the treatment. I then gave her an additional twenty sittings of five minutes with the lupus lamp. 4 gradual improvement set in and the lupus diminished. As it was howerer impossible to bring adequate pressure to bear on the interior and edges of the nostrils, I had again recourse to the $x$-ray treatment and the result was exceedingly satisfactory. There has been no recurrence of the lupus, but owing to a fissure inside one of the nostrils I am giving the patient a few more sittings. The nose is now of ordinary shape, there is no scar to be seeu and no pigmentation, the only change being the acquisition of a rosecoloured tint. (Fig.6)

CASE VII B.-The mother of the former patient developed lupus a year later than her daughter. The situation was the same, but the character of the disease was not so severe. iFig. 7.) The front and sides of the nostrils were affected, and the lupus had extended slightıy up the nostril, where there was some little ulceration. She was accorded the same treatmeut as her duughter with the exception that only a mild reaction took place. She did not attend regularly, howerer, and ceased treatment before I considered her absolutely cured. (Fig. 8.) The result is that she is again under treatment for au infinitesimal spot which will however be eradicated with a few applications.

CASE vill-Boy aged 16. Duration of disease five years. No surgical treatment had been applied. Description: Lupus in front of the riglit ear to the extent of $I$ in. by $\frac{1}{b}$ in. The patient came to me ou August $20 \mathrm{~h}$, 19c2, and was cured by the lupus lamp after eight applications of eight minutes each. This is the most speedy cure effected by me. There has been no recurrence.

CASE 1X.-Woman aged 48. Duration of disease six years. This patient had months later. The affected area was above the tip of the nose, half an inch in extent, and was spreading rapidly (Fig. 9). Commencing April ${ }_{3} \mathrm{rd}$, 1902, I gave her tweuty applications of seven minutes' duration with the lupus lamp, and by the end of May all trace of the disease had 
disappeared, and there was not the slichtest disfigurement (Fig. ro). The patient, who had complained bitterly of neuralgic pains in the diseased parts. was comp] etelyirelievediand restored to health.

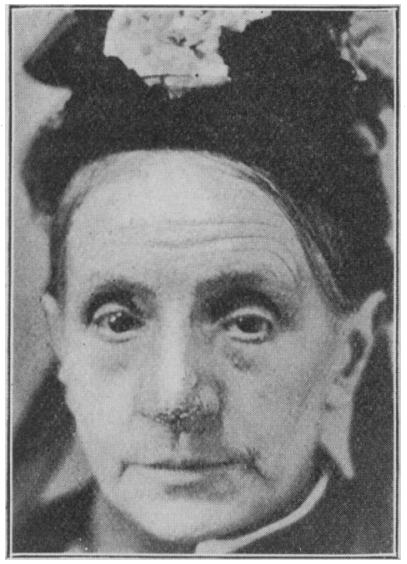

Fig. 7.-Case vil B; condition on April sill, ryoz.

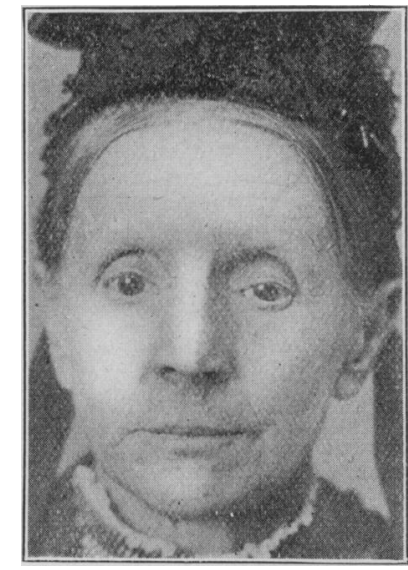
sugust $20 t h$, roo2, after two hours' ar plication of $x$ and ultra-violet
Fig. 8.-Lase vil 13 ; condilion ou

CASE x-Woman, aged 63. Duration of disease fifteen years. Three years after development of diseas the right nostril was thoroughly scraped and cauterized. The patch on the right side of the cheek measured about 2 in. in circumference. Five years ago chloroform was administered and the diseased parts again scraped and catiterized. On May 16th, 1902, the patient came under my treatment. The lupus by this time had spread over the right alae of the nose and $t$ in. into the interior of the nostril. There was also a patch about $\frac{1}{2}$ in. in circumference under the chin. I gave the patient a total of thirty-two applications. After twenty sittings with the lamp of seven minutes' duration the easily-accessible portions on the cheek and underneath the chin disappeared, slow improvement having set in from the outset. The patch on the nose I treated with $x$ rays of about five minutes' exposure; the patient. however, had an obstinate ulceration about $r$ in. up the nostril on the septum. I combated this with a local application of high-frequency current electrode, using a voltage of 36 and an amperagej of 6 . The result was surprisingly rapid. There was no ulceration after five applications. Whether the high-frequency current or the $x$ ray was responsible I cannot definitely state. The scar consequent upon the early operations disappeared, and with the exception of loss of tissue in the situation of the right alae, there was no disfigurement or pigmentation.

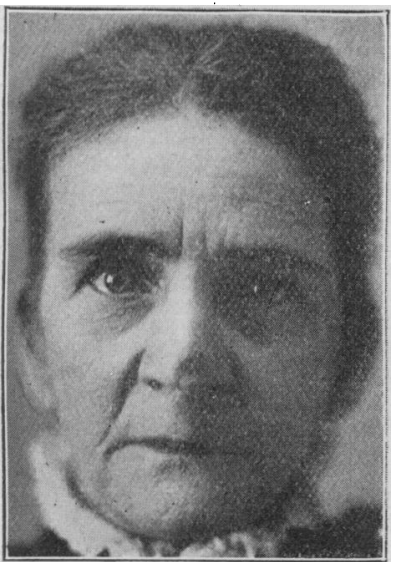

rig. 9.-Case $1 \mathrm{x}$; conaition on April 3 rd, 1902.

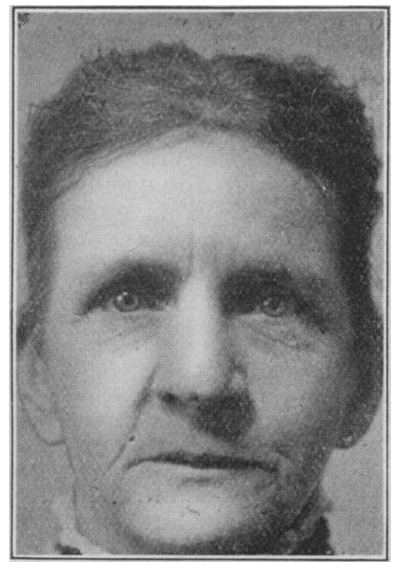
Isth, rgos, after twa hours and twenty minutes
ultra-violet rays.
Fig. 10.-Ciaso IX; condiliou on Jume

CASE XI.-A girl, aged 17. Duration of disease tive years. Early in 1897 the affected part was cauterized, but there was no improvement. The lupus on the nose spread rapidly. The patient was then placed under hospital treatment, and the operation of scraping performed. She was discharged eured after three weeks had elapsed, but a month later the disease recurred. She became an in-patient in another hospital, but the treatment had little, if any, tangible result. Subsequently she was under an eminent specialist in skin diseases, and within three months two operations were performed, the parts being thoroughly scraped. The disease once more recurred, and the patient was under hospital treatment. Another operation had no better results, and she was next sent to a private institution. An operation was performed under chloroform, but again the disease reappeared in a severe form. Later the patient weut to a London skin hospital, and was daily under the $x$-ray treatment ior three months, but returned home at the end of December, rgor. She tirst me to me on August, 23rd, rgo2. (Fig.ri.) Her condition by thi time. was as follows:-Sear over the sides, end, and tip of the nostrils, and extending to part of the upper lip. There was: a) a scar about the size of a five-shilling piece on the right cheek. Dotted here and there over the scar on the nose were fresh tubercles, whilst the same applied to the scar on the richt cheek, especially about the periphery. Some of these were alread $y$ in a state of ulceration. There were also tubercles inside the nostrils and considerable thickening of the bone was noticeable. I gave. the patient a total of twenty $x$-ray sittings. The first five or six applications were of a duration of four minutes each, and I used a very hard tube, giving a spark length of 6 in. to 7 in. I was not satisfied with the progress made, and this prompted me to substitute a soft tube with a $I$ in. spark gap. I also reduced the sittings to two minutes for each part and succeeded in maintaining a mild reaction. Then the nodules on. the nose disappeared fairly rapidly. Recently I prolonged half-a-dozen sittings to a period of five minutes. A severe reaction was the consequence and this development compelled me to give the patient a sitting every third or fourth day instead of every second day as hitherto. At present all the lupus is apparently eradicated and the unsightly scarring

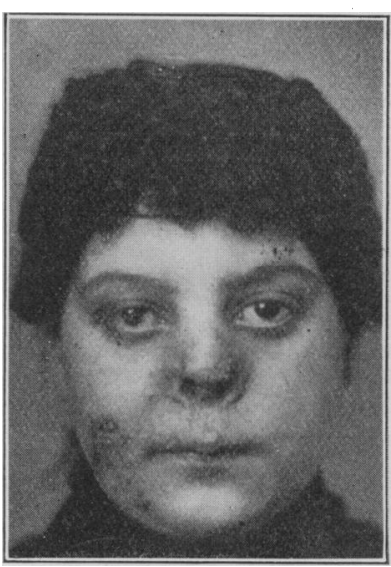

Fig. 11.-Case XI ; cold
August 23 rd, 1902.

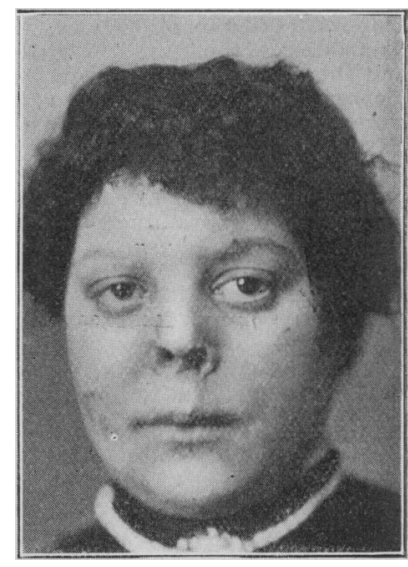

Fis. 12.-Case XI : condition on Janvary 3 rd, 1903. after two hours and is much less pronounced. As a precaution, however, I am giving a few more exposures in order to make the prospect of permanency more definite. The photograph (Fig. 12) shows the condition after cure had been completed.

\section{TUBERCULOSIS OF THE CONJUNCTIVA CURED. BY $X$ RAYS.}

By SYDNEY STEPHENSON, M.B., C.M., F.R.C.S.E., Ophthalmic Surgeon to the North-Eastern Hospital for Children, etc.

TUBERCULOSIS is one of the rarer diseases of the conjunctiva, and, according to $\mathrm{my}$ own experience, fo met with about once in one thousand eye cases seen at a childrer's hospital. It is a chronic process, generally affecting persons under 20 years of age. It often appears to be primary - that is to say, not to be associated with obvious tuberculosis in other parts of the body. This can easily be explained by the exposed position of the conjunctiva, and the frequency of tubercle bacilli in the uir derived from dried phthisical sputum. Foreign bodies, too, as Fuchs supposes, may readily play an important part in the transmission of infection. Then, as more than one writer has painted out, an ulcerated phlyctenule-a condition common enough in the neglected childre $n$ of the poor-may well be the place whereby infection enters the conjunctiva. Lastly, cases of direct operative inoculation have been reported. A family or personal history, however, of tubercle 18 not uncommon' in these cases. It may, indeed; form part of a generally disseminated miliary tuberculosis, as in a case pubiighed by me in the BRITISH MEDICAL JOURNAL of May 3 rd, 1902 . The patient, a female child aged 17 months, suffered 1902. The pationt, a neer of the conjunctiva, with localized destruction of the free edge of the lower lid, a typical tubercle of the choroid, and tabercle of the lungs and of the meninges. The case was fatal.

Tuberulosis of the conjunctiva has several clinical varieties, but it consists essentially, first, in the deposition, and, then, in the disintegration of the tuberculous material in the conjunctiva, palpebral or ocular. Its positive diagnosis implies the liscovery of the bacillus tuberculosis in discharge from the age or in morsels of diseased material removed from the 